\title{
Application of RTK Technology in Vehicle Driving Deviation Test
}

\author{
Xinglin Zhou ${ }^{1, \mathrm{a}}$,Yang $\mathrm{Wu}^{1, \mathrm{~b}}$ and Wensha $\mathrm{Lv}^{1, \mathrm{c}}$ \\ ${ }^{1}$ Wuhan University of Science and Technology, Wuhan, Hubei, China \\ azx165@163.com, ${ }^{b} 591897764 @ q q . c o m,{ }^{c} 852749001 @ q q . c o m$
}

Keywords: Vehicle Driving Deviation; RTK; Measurement Model; Test System

Abstract. This paper presents a method using RTK technology to test the vehicle's driving deviation distance and angle which covers the shortage of the existing method. The measuring principle, composition and algorithm of RTK test system is also introduced in the paper. Two moving receivers were used simultaneously to test the driving deviation of a vehicle at different time periods and different climate conditions. The result of test data comparison showed that RTK method can be used in testing driving deviation. The test system has the characteristic of high speed, effective and high test precision, and it can realize all-weather measurement.

\section{Introduction}

Vehicle driving deviation means that when a vehicle is driving straight-line at a high speed on a flat surface, the vehicle turns to deviate to the left or right after taking the hands off the wheel. Potential problems of vehicle driving deviation range from driving fatigue to traffic accident. It can also cause uneven wear or even ruin the tire.

In our country, the research to the vehicle driving deviation test is still in the stage of exploration and development. A comprehensive vehicle driving deviation test system hasn't been developed. Some of the existing methods have low test accuracy, and some are easily affected by environment so that they can't be used around the clock. It is necessary to develop a fast and efficient method to test the vehicle driving deviation distance and angle.

\section{Test model}

\section{Working principle of RTK}

RTK (Real Time Kinematic) is real-time differential GPS technology based on carrier phase observations. RTK system usually consists of three parts: base station, rover station and data communication chain. The principle is set a base station at a known spot, the station keeps observing the satellites and its observation data and station information are sent to the rover station through the radio transmission equipment. The rover station constitutes a difference algorithm by receiving the information from the base station and the satellites. Then high-precision coordinates of rover station are given. Carrier phase differencing technique can provide centimeter-level position accuracy within the scope of the $40 \mathrm{~km}$.

\section{Testing system modeling}

As shown in Fig. 1, the base station 1 is set beside the test road, it observes the satellites for a period of time and the average of satellite observation can be used as base station reference coordinates. The rover station 3 is fixed on the top of the test vehicle 2 . In the beginning the steering wheel should be controlled to accelerate the vehicle to the test speed along the road center-line, then loosen the steering wheel and keep the test speed until driving out of the test area. The trajectory information of the test vehicle is recorded by the rover station. 


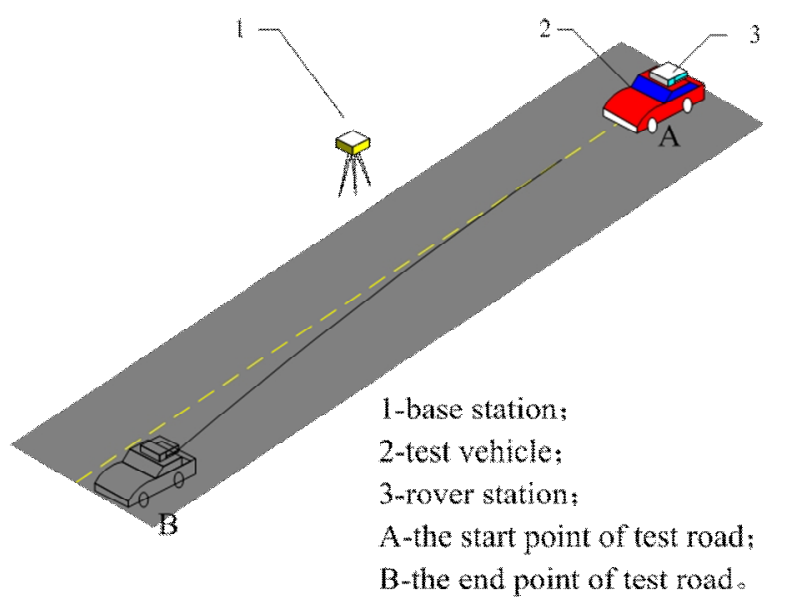

Fig. 1 Vehicle driving Deviation measurement model

\section{Calculation model}

WGS-84 coordinate system is a geodetic coordinate system whose origin is the center of earth. It can't be directly used to calculate the driving deviation distance and angle. So we need to convert the recorded data into space rectangular coordinate, the coordinate conversion formulas are as follows:

(1)

$$
x=(N+H) * \cos B * \cos L
$$

$$
\begin{aligned}
& y=(N+H) * \cos B * \sin L \\
& z=\left[N\left(1-e^{2}\right)+H\right] * \sin B \\
& \mathrm{~N}=\frac{a}{\sqrt{1-e^{2} \sin ^{2} B}}
\end{aligned}
$$

Where $\mathrm{B}$ is the latitude of detecting point, and $\mathrm{L}$ is the longitude of detecting point, and $\mathrm{H}$ is the elevation of detecting point, and a is the major axis semidiameter of ellipsoid in WGS-84 coordinate system $(\mathrm{a}=6378137.00 \mathrm{~m})$, and $\mathrm{e}^{2}$ is the first eccentricity of WGS-84 coordinate system $\left(\mathrm{e}^{2}=0.0066943799013\right)$.

The converted coordinates are shown in Fig. 2. $\mathrm{AB}$ is the traveling track of test vehicle. The coordinate points recorded by the rover station are named $\left(X_{1}, Y_{1}, Z_{1}\right),\left(X_{2}, Y_{2}, Z_{2}\right) \ldots\left(X_{n}, Y_{n}, Z_{n}\right)$ in turn. Due to the high sampling rate, the traveling track between two points can be considered as the straight line connects the two points. The vehicle trajectory equation is shown in Eq. 5 .

$$
\left\{\begin{array}{l}
\frac{X-X_{1}}{X_{2}-X_{1}}=\frac{Y-Y_{1}}{Y_{2}-Y_{1}}=\frac{Z-Z_{1}}{Z_{2}-Z_{1}} \quad\left(X_{1}<X<X_{2}\right) \\
\frac{X-X_{2}}{X_{3}-X_{2}}=\frac{Y-Y_{2}}{Y_{3}-Y_{2}}=\frac{Z-Z_{2}}{Z_{3}-Z_{2}} \quad\left(X_{2}<X<X_{3}\right) \\
\mathrm{M} \\
\frac{X-X_{n-1}}{X_{n}-X_{n-1}}=\frac{Y-Y_{n-1}}{Y_{n}-Y_{n-1}}=\frac{Z-Z_{n-1}}{Z_{n}-Z_{n-1}} \quad\left(X_{n}<X<X_{n-1}\right)
\end{array}\right.
$$




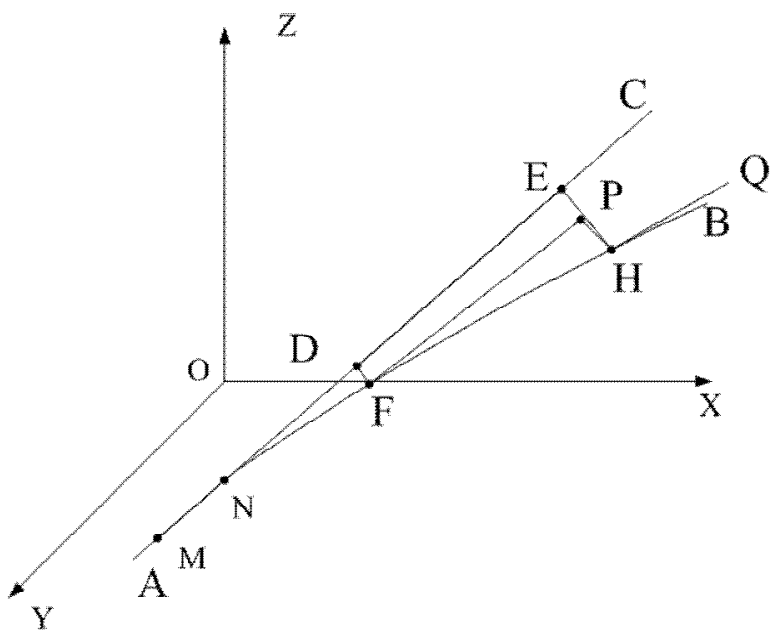

Fig. 2 The traveling track of test vehicle

$\mathrm{M}\left(\mathrm{X}_{\mathrm{M}}, \mathrm{Y}_{\mathrm{M}}, \mathrm{Z}_{\mathrm{M}}\right), \mathrm{N}\left(\mathrm{X}_{\mathrm{N}}, \mathrm{Y}_{\mathrm{N}}, \mathrm{Z}_{\mathrm{N}}\right)$ are any two points on road axis that are also on the track $A B$, the equation of road center line (AC) can be calculated according to $M$ and $N$. Find two points $D\left(X_{D}, Y_{D}\right.$, $\left.\mathrm{Z}_{\mathrm{D}}\right)$ and $\mathrm{E}\left(\mathrm{X}_{\mathrm{E}}, \mathrm{Y}_{\mathrm{E}}, \mathrm{Z}_{\mathrm{E}}\right)$ on the road center line that correspond to the vehicle deviation track and the distance of $\mathrm{DE}$ is 100 meters. The equations of the plane across point $\mathrm{D}$ and perpendicular to line $\mathrm{AC}$ and the plane across point $\mathrm{E}$ and perpendicular to line $\mathrm{AC}$ can be calculated. The former is named plane $l$ and the latter is named plane $\mathrm{m}$.

The coordinate of $\mathrm{F}\left(\mathrm{X}_{\mathrm{F}}, \mathrm{Y}_{\mathrm{F}}, \mathrm{Z}_{\mathrm{F}}\right)$, the point on the vehicle deviation track that corresponds to point $\mathrm{D}$, can be calculated by combining Eq. 5 and plane 1. The equation of the line that $F$ lies on is:

$$
\frac{X-X_{f}}{X_{f+1}-X_{f}}=\frac{Y-Y_{f}}{Y_{f+1}-Y_{f}}=\frac{Z-Z_{f}}{Z_{f+1}-Z_{f}} \quad\left(X_{f}<X<X_{f+1}\right)
$$

The coordinate of $\mathrm{H}\left(\mathrm{X}_{\mathrm{H}}, \mathrm{Y}_{\mathrm{H}}, \mathrm{Z}_{\mathrm{H}}\right)$, the point on the vehicle deviation track that corresponds to point $\mathrm{E}$, can be calculated by combining Eq. 5 and plane $\mathrm{m}$. The equation of the line that $\mathrm{H}$ lies on is:

$$
\frac{X-X_{h}}{X_{h+1}-X_{f}}=\frac{Y-Y_{h}}{Y_{h+1}-Y_{f}}=\frac{Z-Z_{h}}{Z_{h+1}-Z_{h}} \quad\left(X_{h}<X<X_{h+1}\right)
$$

FP is tangent to the vehicle deviation track at point F. HP is perpendicular to FP. HQ is tangent to the vehicle deviation track at point $\mathrm{H}$. Then the distance between $\mathrm{H}$ and FP is the one hundred meters deviation distance $(\Delta \mathrm{L})$, and the included angle between FP and HQ is the one hundred meters deviation angle $(\Delta \theta)$. The formulas are:

$$
\begin{aligned}
& \Delta \mathrm{L}=H P=\frac{\sqrt{\left|\begin{array}{cc}
Y_{H}-Y_{F} & Z_{H}-Z_{F} \\
Y_{f+1}-Y_{f} & Z_{f+1}-Z_{f}
\end{array}\right|+\left|\begin{array}{cc}
Z_{H}-Z_{F} & X_{H}-X_{F} \\
Z_{f+1}-Z_{f} & X_{f+1}-X_{f}
\end{array}\right|+\left|\begin{array}{cc}
X_{H}-X_{F} & Y_{H}-Y_{F} \\
X_{f+1}-X_{f} & Y_{f+1}-Y_{f}
\end{array}\right|}}{\sqrt{\left(X_{f+1}-X_{f}\right)^{2}+\left(Y_{f+1}-Y_{f}\right)^{2}+\left(Z_{f+1}-Z_{f}\right)^{2}}} \\
& \Delta \theta=\frac{\left(X_{f+1}-X_{f}\right)\left(X_{h+1}-X_{h}\right)+\left(Y_{f+1}-Y_{f}\right)\left(Y_{h+1}-Y_{h}\right)+\left(Z_{f+1}-Z_{f}\right)\left(Z_{h+1}-Z_{h}\right)}{\sqrt{\left(X_{f+1}-X_{f}\right)^{2}+\left(Y_{f+1}-Y_{f}\right)^{2}+\left(Z_{f+1}-Z_{f}\right)^{2}} \sqrt{\left(X_{h+1}-X_{h}\right)^{2}+\left(Y_{h+1}-Y_{h}\right)^{2}+\left(Z_{h+1}-Z_{h}\right)^{2}}}
\end{aligned}
$$




\section{The composition of the test system}

The main hardware of the test system is shown in Fig. 3. The system includes three parts: data acquisition module, data processing module and wireless communication module. RTK module is used to collect the track information of the test vehicle by observing satellites. In addition to data processing, the computer is also used to control the RTK module. The handhold terminal is used to display the vehicle driving deviation distance and angle, and the radio station and the wireless access point is used to transmit data.

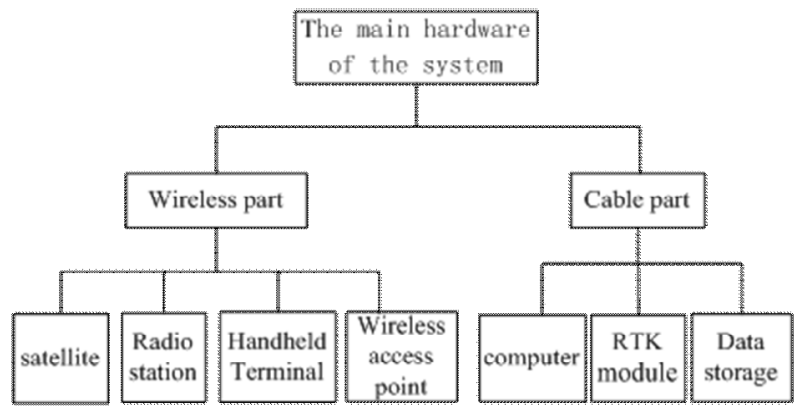

Fig. 3 The main hardware of the system

In order to do the detection, the RTK module should be set up first. The computer can be used to debug the RTK module and record the VIN of the vehicle. The test will start after ensuring all equipment is working properly. RTK module records the traveling track of the test vehicle throughout the entire process. At the same time, the rover receiver transmits the data to the computer continuously. After calculating the vehicle driving deviation distance and angle, the computer will pass the result to the handhold terminal through wireless access point. If the test will continue, just repeat the steps above.

\section{Example analysis}

In order to test and verify the accuracy and reliability of the system, set up a base station and two of the same rover station. Installed the two rover station on the same test vehicle, and tested the vehicle several times at the speed of $40 \mathrm{~km} / \mathrm{h}$, calculated the one hundred meters deviation distance and angle of the vehicle using the data received by the two rover stations separately. Then contrast the result to demonstrate the feasibility and effectiveness of theoretical research. GNSS receiver shown in Fig. 4 was used in this system. It was produced by NovAtel Company. The main technical parameters of the receiver are shown in table 1 . The following is the result of example analysis.

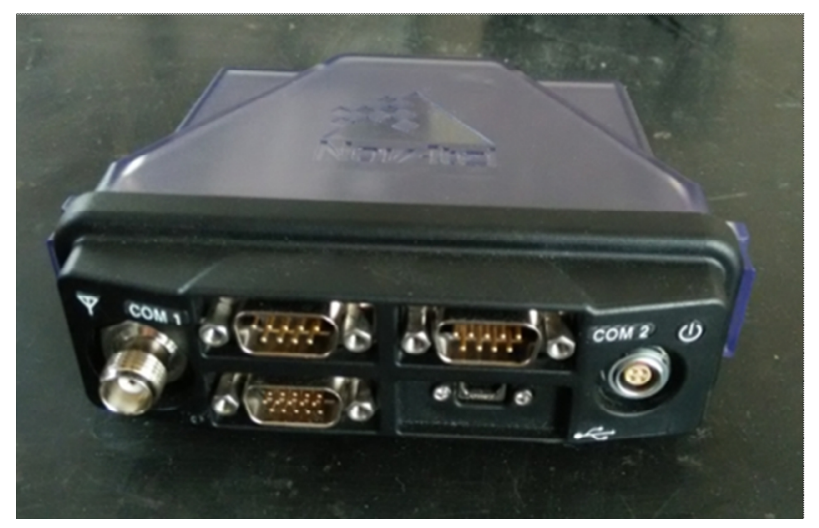

Fig. 4 The GNSS receiver 
Table 1 The main technical parameters of the receiver

\begin{tabular}{c|c}
\hline Performance Indicators & Precision \\
\hline Real time positioning precision & $1 \mathrm{~cm}+1 \mathrm{ppm}$ \\
\hline Velocity-measuring precision & $\leq 0.03 \mathrm{~m} / \mathrm{s}$ \\
\hline Speed dynamic indicator & $\leq 515 \mathrm{~m} / \mathrm{s}$ \\
\hline Maximum sampling frequency & $20 \mathrm{~Hz}$ \\
\hline
\end{tabular}

\section{Device debugging}

Set up the base station, two points A and B was demarcated on the road. The two rover receivers were installed at point $A$ and $B$ separately to do the repeatability test to validate the repeat-positioning veracity of the receivers. The result is shown in Table 2.

Table 2 Repeatability test result of the system

\begin{tabular}{c|c|c|c|c|c|c}
\hline \multirow{2}{*}{$\begin{array}{c}\text { Testing } \\
\text { number }\end{array}$} & \multicolumn{3}{|c|}{ Receiver 1 in point A } & \multicolumn{3}{c}{ Receiver 2 in point B } \\
\cline { 2 - 7 } & $\mathrm{X}[\mathrm{m}]$ & $\mathrm{y}[\mathrm{m}]$ & $\mathrm{z}[\mathrm{m}]$ & $\mathrm{x}[\mathrm{m}]$ & $\mathrm{y}[\mathrm{m}]$ & $\mathrm{z}[\mathrm{m}]$ \\
\hline 1 & 3435171.902 & 326065.0440 & 137.4985 & 3435178.815 & 326063.8836 & 137.1144 \\
\hline 2 & 3435171.902 & 326065.0440 & 137.4971 & 3435178.815 & 326063.8836 & 137.1161 \\
\hline 3 & 3435171.904 & 326065.0441 & 137.4966 & 3435178.817 & 326063.8836 & 137.1155 \\
\hline 4 & 3435171.900 & 326065.0440 & 137.4957 & 3435178.815 & 326063.8836 & 137.1169 \\
\hline 5 & 3435171.902 & 326065.0440 & 137.4963 & 3435178.812 & 326063.8835 & 137.1157 \\
\hline 6 & 3435171.904 & 326065.0441 & 137.4982 & 3435178.810 & 326063.8676 & 137.1133 \\
\hline 7 & 3435171.904 & 326065.0441 & 137.4967 & 3435178.812 & 326063.8835 & 137.1131 \\
\hline 8 & 3435171.906 & 326065.0441 & 137.4946 & 3435178.812 & 326063.8835 & 137.1138 \\
\hline 9 & 3435171.906 & 326065.0441 & 137.4967 & 3435178.812 & 326063.8676 & 137.1169 \\
\hline 10 & 3435171.904 & 326065.0441 & 137.4956 & 3435178.812 & 326063.8676 & 137.1148 \\
\hline$\sigma$ & 0.0018 & 0.00004899 & 0.001110 & 0.002039608 & 0.007312599 & 0.001322 \\
\hline
\end{tabular}

As seen in Table 2, the root-mean-square errors of the two receivers in repeatability test are all within $1 \mathrm{~cm}$. So the receivers had a relatively good repeatability.

\section{Detection result}

Installed the two rover station on the test vehicle after debugging the system and tested the vehicle. The track of the vehicle recorded by receiver 1 and receiver 2 is shown in Fig. 5. It was calculated that the one hundred meters deviation distance test by receiver 1 was $58.63454 \mathrm{~cm}$, and the angle was $0.365484873^{\circ}$. The one hundred meters deviation distance test by receiver 2 was $58.68546 \mathrm{~cm}$, and the angle was $0.369845296^{\circ}$.

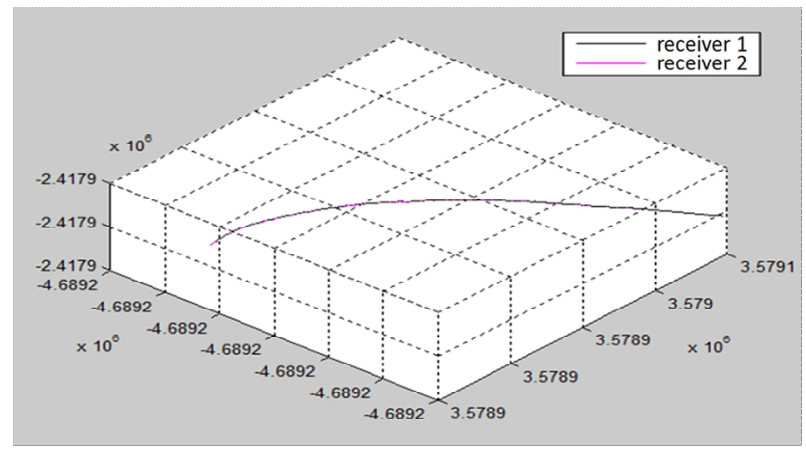

Fig. 5 The track of the vehicle recorded by receiver 1 and receiver 2

Use the same method to test the vehicle at different times and different climate conditions. The result is shown in Table 3. 
Table 3 Test result of the two rover receivers

\begin{tabular}{|c|c|c|c|c|}
\hline \multirow[b]{2}{*}{$\begin{array}{l}\text { Testing } \\
\text { number }\end{array}$} & \multicolumn{2}{|c|}{ Receiver 1} & \multicolumn{2}{|c|}{ Receiver 2} \\
\hline & $\begin{array}{c}\text { One hundred meters } \\
\text { deviation } \\
\text { distance }[\mathrm{cm}]\end{array}$ & $\begin{array}{c}\text { One hundred meters } \\
\text { deviation angle }\left[{ }^{\circ}\right]\end{array}$ & $\begin{array}{c}\text { One hundred meters } \\
\text { deviation } \\
\text { distance }[\mathrm{cm}]\end{array}$ & $\begin{array}{c}\text { One hundred meters } \\
\text { deviation angle }\left[{ }^{\circ}\right]\end{array}$ \\
\hline 1 & 58.90524 & 0.356518977 & 58.76245 & 0.355943728 \\
\hline 2 & 57.79479 & 0.358364474 & 57.90254 & 0.358015543 \\
\hline 3 & 58.50988 & 0.356358549 & 58.36947 & 0.356298389 \\
\hline 4 & 57.91074 & 0.356723523 & 57.86479 & 0.356741285 \\
\hline 5 & 57.93913 & 0.359224484 & 57.89645 & 0.357209391 \\
\hline 6 & 59.03912 & 0.356227915 & 58.93578 & 0.356224477 \\
\hline 7 & 58.82669 & 0.356033682 & 58.59314 & 0.357438002 \\
\hline 8 & 58.42473 & 0.358383382 & 58.49977 & 0.360341179 \\
\hline 9 & 59.10535 & 0.355979251 & 59.21023 & 0.356948696 \\
\hline 10 & 58.79598 & 0.356120199 & 58.76587 & 0.356162025 \\
\hline
\end{tabular}

According to the deviation distance and angle tested by the two receivers in table 3, the difference curve can be drawn in Fig. 6 and Fig. 7. It can be seen that the one hundred meters deviation distance difference is within $1 \mathrm{~cm}$ and the one hundred meters deviation angle difference is within $0.003^{\circ}$. It can be proved that the test system operated stable and reliable with a high detection precision. It can meet the needs of production line testing.

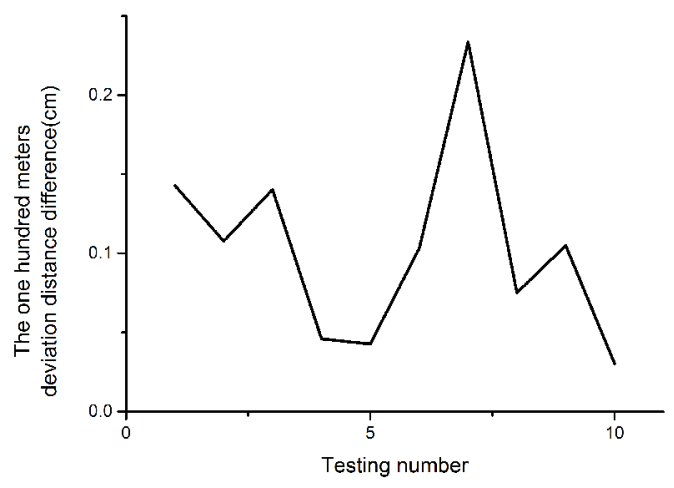

Fig. 6 The one hundred meters deviation distance difference curve

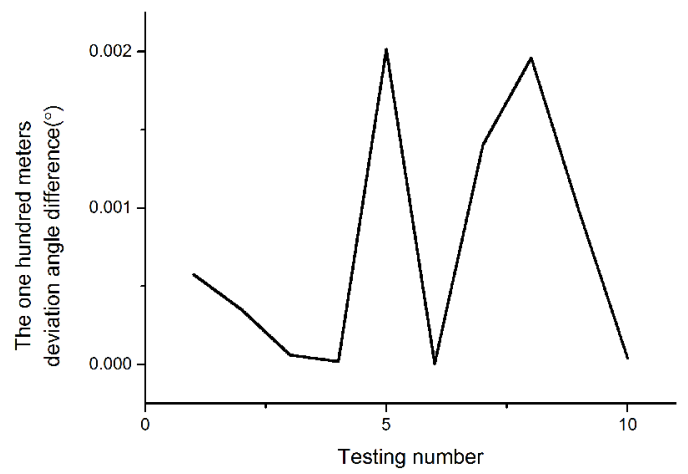

Fig. 7 The one hundred meters deviation angle difference curve

\section{Conclusion}

This paper proposed a vehicle running deviation detection method using RTK method. A 
three-dimension model of vehicle traveling track was set up, and the computational formulas of one hundred meters driving deviation distance and angle was given in the paper. Comparing to convert the WGS-84 coordinates to the plane coordinates directly; three-dimension method had a higher precision. It was proved through comparison experiments that the system could realize all-weather vehicle deviation test with a high precision, and the system operated stable and reliable. It can be widely applied in vehicle production line testing, and had practical significance for vehicle deviation test. It also provided a new idea for the test of vehicle deviation.

\section{Acknowledgements}

This work was financially supported by the Natural Science Foundation of China (51578430), Science and Technology Support Project of Hubei province (2014BEC055) and Nature Science Foundation of Hubei Province (2015CFA064).

\section{References}

[1] Xinglin Zhou, Cheng Li, Hanli Liu, et al. On-line Automatic Vehicle Driving Wandering Test System Based on Binocular Vision [J]. China Mechanical Engineering, 2015, (1): 130-134.

[2] Yaohua He, Can Yang, et al. Vehicle Driving Wandering Test System Based on LabVIEW [C]. 2009 SAE-China Congress PROCEEDINGS. 2009: 766-769.

[3] Changjiang An, Wenyong Li. A Study on the Measurement Methods of Vehicle Driving Deviation Based on GPS Technology [J]. Automotive Engineering. 2009, 31(9): 804-806.

[4] Yaohua He, Xiaofei Li. Vehicle-wandering testing system based on closerange photogrammetric technology [J]. Chinese Journal of Construction Machinery. 2011, 09(4): 476-481.

[5] Moon Y G, Kim M S, Lee M C. Development of Autonomous Vehicle Control Algorithm Based on DGPS(RTK) and Test Vehicle Performance Verification [J]. ICCAS 2012, 2012: 1644-1648.

[6] Moon Y G, Kim M S, Lee M C. Development of speed control and path following algorithm based on RTK-DGPS [J]. ICCAS 2011, 2011, 86(1): 734-737.

[7] Siguang Fan. Performance Test Technology in Vehicle Based on GPS [J]. Automobile Parts. 2012, (1): 88-91.

[8] Wuhan University of Technology. On-line Automatic Inspection System of Vehicle Driving Wandering Based on Optical Fiber Sensing: China, CN201110039758.2 [P]. 2011-9-14.

[9] Jie Ke. Testing System of the Vehicle Driving Wandering Based on Fiber Bragg Grating Sensor [D]. Wuhan University of Technology. 2012.

[10] Shujuan Cui. Image Processing Technology Research of Vehicle Running Deviation Online Automatic Detection System [D]. Wuhan University of Technology. 2010.

[11] Chuqi Su, Libo Huang. Automatic Inspection of Vehicle Running Deviation Based on Image Processing [J]. Tianjin Auto, 2008, (11): 46-48.

[12] Can Yang. Vehicle Driving Wandering Test System Based on LabVIEW Research and Development [D]. Wuhan University of Technology. 2010. 